Poster (C24)

Cervical Cancer

https://doi.org/10.3802/jgo.2021.32.S1.C24

\section{Treatment patterns and surgical outcome of stage IA1 cervical carcinoma}

\author{
Charuwan Saeteng, Unyavee Apichottiwat, \\ Kittipat Charoenkwan, Prapaporn Suprasert, \\ Jatupol Srisomboon \\ Chiang Mai University, Chiang Mai, Thailand (nokctantipa@gmail.com)
}

Objective: To evaluate treatment patterns and surgical outcome of stage IA1 cervical cancer.

Methods: Patients with stage IA1 cervical cancer diagnosed between January 2001 and June 2018 at Chiang Mai University Hospital were retrospectively reviewed. The analysis included treatment patterns, surgical types, and clinicopathologic variables, i.e., nodal metastasis, parametrial involvement, positive surgical margins, deep stromal invasion, lymph-vascular space invasion, adjuvant treatment, and 5-year disease-free survival and 5-year overall survival. All pathologic slides were reviewed by gynecologic pathologists. Kruskal-Wallis test and Fisher's exact test, KaplanMeier method, and log-rank test were used for statistical analysis. Results: One-hundred eighty-five patients were included in this study. Simple hysterectomy was the major treatment $(57.3 \%)$ followed by modified radical hysterectomy and radical hysterectomy (27.6\% and $9.7 \%$ respectively). Conization and radiation were chosen in few cases. At the median follow-up time of 40.8 months, the 5-year disease-free survival rate was $99.1 \%$ and the 5-year overall survival rate was 95.0\%. Pelvic lymph node dissection was done in 62 cases (33.7\%), but only one $(0.54 \%)$ had pelvic lymph node metastasis.

Conclusion: Surgical and survival outcomes of women with stage IA1cervical cancer are excellent. These could be effectively treated by conservative treatment (simple hysterectomy and conization). Lymph node metastasis is rare in this stage, therefore, the lymphadenectomy could be omitted.

Poster (C25)

Gynecologic Cancer Screening

https://doi.org/10.3802/jgo.2021.32.S1.C25

\section{Quality assurance for the cervical cancer screening program in Japan}

Kanako Kono, ${ }^{1}$ Kumiko Saika, ${ }^{2}$ Eiko Saitoh, ${ }^{3}$ Tomio Nakayama, ${ }^{4}$ Tohru Morisada, ${ }^{5}$ Daisuke Aoki ${ }^{1, *}$

'Department of Obstetrics and Gynecology, Keio University School of Medicine, Tokyo, Japan (aoki@z7.keio.jp)
${ }^{2}$ Division of International Health Policy Research, National Cancer Center, Tokyo, Japan

${ }^{3}$ Preventive Medicine Center, International University of Health and Welfare, Mita Hospital, Tokyo, Japan

${ }^{4}$ Division of Screening Assessment and Management, National Cancer Center, Tokyo, Japan

${ }^{5}$ Department of Obstetrics and Gynecology, Faculty of Medicine, Kyorin University, Tokyo, Japan

Objective: Cervical cancer screening has been implemented nationwide since 1982 in Japan and managed by 1,737 local governments (LGs). In order to improve the level of quality assurance (QA), the Ministry of Health, Labour and Welfare (MHLW) released a guide in 2007 which contains QA instructions and implementation procedures. The aim of this study is to introduce the indicators for the QA structure in the cervical cancer screenings.

Methods: The MHLW and National Cancer Center (NCC) play roles in formulating the screening program's QA. We obtained cancer screening indicators from the NCC's website and MHLW's portal site which pertained to the QA structure.

Results: The guide described 2 main indicators for QA: one is a compliance rate using checklists which listed 50 structural criteria for conducting screening, and the other is a process indicator which is detailed screening results. NCC surveys compliance rate in each LG and publishes it as quality control indicators on their website. Regarding process indicators, all LGs submit annual screening results including the number of women who underwent screening, screen negative, referral, and detection to diagnose not only the cervical cancer but also cervical intraepithelial neoplasia (CIN) 3, CIN2, and CIN1 to the MHLW and they published detailed screening results by age group sand screening histories. Conclusion: The cancer screening programs established QA structures based upon compliance rates and process indicators, however comprehensive evaluation strategies need to be formulated for evaluating the screening activities in order to detect problematic issues and to improve QA.

Poster (C26)

Cervical Cancer

https://doi.org/10.3802/jgo.2021.32.S1.C26

\section{Active clinical trials of cervical cancer committee in KGOG}

Yong Wook Jung, ${ }^{1} \mathrm{Ju}$ Won Roh, ${ }^{2}$ Kwang Beom Lee, ${ }^{3}$ Min-Hyun Baek, ${ }^{4}$ Ki Dong Kim, ${ }^{5}$ Keun Ho Lee ${ }^{6, *}$

${ }^{1} \mathrm{CHA}$ Gangnam Medical Center, CHA University, Seoul, Korea ${ }^{2} \mathrm{CHA}$ Ilsan Medical Center, CHA University, Goyang, Korea ${ }^{3}$ Gachon University Gil Medical Center, Incheon, Korea ${ }^{4}$ Hallym University Sacred Heart Hospital, Anyang, Korea ${ }^{5}$ Seoul National University Bundang Hospital, Seongnam, Korea ${ }^{6}$ Seoul St. Mary's Hospital, The Catholic University of Korea, Seoul, Korea (HOHOHO@CATHOLIC.AC.KR) 
Background: Clinical trials are important to develop new treatment and disease prevention strategy for cervical cancer. Cervical Cancer Committee of Korean Gynecologic Oncology Group (KGOG) have been conducting several clinical trials to contribute to overcome the uterine cervical cancer. We would like to briefly introduce our efforts in this presentation. Methods: Cervical Cancer Committee of KGOG is conducting 8 clinical trials including 3 international collaborating studies, 2 prospective clinical trials and 3 retrospective studies. Two international studies were as follows: 1) KGOG-1008 is collaborating study with GOG-263: Randomized phase III clinical trial of adjuvant radiation vs. chemoradiation in intermediate risk, stage I/IIA cervical cancer treated with initial radical hysterectomy and pelvic lymphadenectomy; 2) KGOG1023 is a joint study with RTOG-0724: Phase III randomized study of concurrent chemotherapy and pelvic radiation therapy with or without adjuvant chemotherapy in high-risk patients with early-stage cervical carcinoma following radical hysterectomy. Following 2 clinical trials are being conducted independently by KGOG: 1) KGOG-1036: Prospective-specimencollection and retrospective blinded-evaluation study of human papillomavirus (HPV) viral load to improve cervical cancer screening. The purpose of KGOG-1036 is to examine whether genotype specific quantitative HPV testing can predict the progress of low grade squamous intraepithelial lesion in uterine cervix into high grade lesion requiring treatment; 2) KGOG-1038: Phase II study of belotecan monotherapy in patients with recurrent or persistent cervical cancer. The aim of KGOG-1038 is to demonstrate the objective response rate of belotecan monotherapy for the treatment of recurrent or persistent cervical cancer. The study is recruiting 21 subjects with recurrent or persistent cervical cancer. In addition, 2 retrospective studies are ongoing: 1) KGOG-1037: Impact of metformin medication on survival outcome of cervical cancer. The study is to examine the impact of metformin medication on the prognosis of cervical cancer in patients with diabetes mellitus; 2) KGOG-1042: Prediction of ovarian transposition in young patients with early-stage cervical cancer undergoing surgery (PRETTY): a Korean multicenter retrospective study. The prediction model developing.

Poster (C27)

Cervical Cancer

https://doi.org/10.3802/jgo.2021.32.S1.C27

\section{Ovarian transposition and cervical cancer}

Ingporn Jiamset, Kulisara Nanthamongkolkul, Rakchai Buhachat*

Prince of Songkla University, Songkhla, Thailand (brakchai@yahoo.com)
Transposition of ovaries remains a basic surgical technique to preserve ovarian function for fertility outcome and hormonal status with acceptable risk for metastases in premenopausal cervical cancer. Cervical cancer is a major gynecologic malignancy worldwide. In early-stage cervical cancer, surgery is the main treatment, opposite to the locoregional stage or metastatic disease that concurrent chemoradiation remains the mainstay management. Premature ovarian insufficiency in irradiated field leads to cardiovascular morbidity, osteoporosis, sexual dysfunction, infertility, poor psychological well-being and a reduced quality of life. Advanced cervical cancers unfortunately occurred in younger women with longer survival made the ovarian transposition to be indicated. Transposition of ovaries aims to place the ovaries outside the irradiated field in order to diminish the exposure to radiation and total dose of irradiation. We present the laparoscopic ovarian transposition with suture and clip in a 20-year-old woman with cervical cancer stage IIB.

\section{Uterine corpus cancer}

Poster (E01)

Endometrial Hyperplasia, Endometrial Intra-epithelial Neoplasia, and Endometrial Cancer

https://doi.org/10.3802/jgo.2021.32.S1.E01

\section{Adjuvant chemotherapy alone compared with chemotherapy plus radiotherapy for stage III endometrial carcinoma}

\author{
Tipaya Detpittayanan, Atthapon Jaishuen* \\ Siriraj Hospital, Bangkok, Thailand (ajaishuen@hotmail.com).
}

Objective: To compare treatment outcomes of adjuvant chemotherapy alone (CMT) and adjuvant chemotherapy followed by radiotherapy (CMT/RT) for stage III endometrial carcinoma.

Methods: Medical record of 211 patients with stage III endometrial cancer (International Federation of Gynecology and Obstetrics 2009) who underwent surgical staging at Faculty of Medicine Siriraj Hospital, Mahidol University during 2007-2019 were retrospective reviewed. Patients received either adjuvant CMT or adjuvant CMT/RT. Treatment outcomes between 2 groups of patients were compared at 2 years. Results: There were 123 patients received adjuvant CMT and 88 patients received adjuvant CMT/RT. Baseline characteristics such as age, body mass index, pathology were not different between both 2 groups. Most patients in CMT group had stage IIIA (41.5\%), while most patients in CMT/RT group had 